\title{
An interactive teaching device simulating intussusception reduction
}

\author{
Rebecca Stein-Wexler • Thomas Sanchez • Glade E. Roper • Anthony S. Wexler • \\ Robert P. Arieli • Clark Ho • Joseph C. Li • Alp Ozpinar • Steffan K. Soosman
}

Received: 27 February 2010 / Revised: 16 June 2010 / Accepted: 17 June 2010 /Published online: 21 July 2010

(C) The Author(s) 2010. This article is published with open access at Springerlink.com

\begin{abstract}
Intussusception is relatively uncommon, occurring in 0.5 to 2.3 cases per 1,000 live births in the USA. Radiology residents, therefore, have few opportunities to participate in intussusception reduction during training, and practicing radiologists encounter it infrequently. Training is essential, as successful reduction avoids surgery. The judgment involved in reducing an intussusception is best gained with experience. We developed a training device that simulates fluoroscopic intussusception reduction with air. The device consists of a doll that contains a cylinder with similar stress and strain characteristics to the human colon. The trainee pumps air into the cylinder through a rectal tube using a standard hand-held air reduction pump. A sensor measures the pressure within the chamber and transmits readings to a computer, which displays images from actual intussusception reductions based on the pressure maintained within the device. A random compo-
\end{abstract}

Electronic supplementary material The online version of this article (doi:10.1007/s00247-010-1764-x) contains supplementary material, which is available to authorized users.

R. Stein-Wexler $(\bowtie) \cdot$ T. Sanchez $\cdot$ G. E. Roper

Department of Radiology, University of California at Davis,

4860 Y St., Ste. 3100 ,

Sacramento, CA 95817, USA

e-mail: rebecca.steinwexler@ucdmc.ucdavis.edu

\section{A. S. Wexler}

Department of Mechanical and Aerospace Engineering, Civil and Environmental Engineering, Land, Air, and Water Resources, University of California at Davis, Davis, CA, USA

R. P. Arieli • C. Ho · J. C. Li • A. Ozpinar · S. K. Soosman Department of Biomedical Engineering,

University of California at Davis,

Davis, CA, USA nent in the software gives the user a new experience each time and models uncertainties in the actual reduction process, including perforation. This intussusception reduction simulator can enhance resident education, giving residents the opportunity to practice this technique before employing it on a real patient. The simulator can also help practicing radiologists become more comfortable with intussusception air reduction.

Keywords Intussusception - Reduction · Teaching model · Education $\cdot$ Simulation $\cdot$ Children

\section{Introduction}

Intussusception is an important cause of acute abdomen in infancy and childhood. The incidence of intussusception is estimated to be $0.5-2.3$ cases per 1,000 live births in the USA [1, 2]. However, it is likely that the incidence is actually higher, as some cases resolve spontaneously before being confirmed either radiographically or surgically [3]. The relative rarity of this condition limits radiology residents' opportunity to gain supervised experience in the non-surgical reduction of intussusception. A recent survey found that $22 \%$ of senior radiology residents have had no experience in reducing intussusception, while $21 \%$ have been involved with only one case during their entire residency [4]. However, Meyer et al. [5] demonstrated that a radiologist must be involved in at least four air intussusception reductions to become skillful with this technique.

Nonsurgical reduction of intussusception is the preferred method of treatment, and both air and liquid reduction have been shown to be safe and effective [6]. Hence, it falls squarely on the radiologist to be familiar with and 
proficient at performing this procedure so that patients avoid unnecessary surgery. Since the judgment, confidence and skill involved in reducing intussusception are best gained with experience, radiology residents - and any other practicing radiologists who might be asked to treat a patient's intussusception-will benefit from the training tool we describe here to supplement their caseload. Simulation tools such as this intussusception reduction simulator constitute a major revolution in medical education [7].

\section{Description}

This was a collaborative effort among radiologists and both student and faculty engineers at the UC Davis College of Engineering and was reviewed and approved by our institution's IRB. We developed an interactive teaching device to simulate reducing an intussusception with air under the guidance of an instructor. During the simulation, the instructor controls the ease of maintaining pressure within the device and thus the speed of the reduction, while coaching the trainee. The interaction between the instructor and the trainee is essential to the learning process.

Our goals in developing this teaching device were to:

(1) Help the trainee recognize the progression and reduction of intussusception.

(2) Simulate complications such as perforation that the trainee should detect immediately.

(3) Monitor the fluoroscopy time with the intention of improving technique to lower radiation dose.

(4) Allow the instructor to control the trainee's success at maintaining adequate pressure, so that the trainee develops awareness of the importance of the rectal "seal."

(5) Create a compact, portable device that can be easily assembled and that has a user-friendly interface for both trainee and instructor.

The resulting device (Fig. 1, "Lucy") consists of an airtight cylindrical chamber made from polyvinyl chloride pipe, housed inside a baby doll and accessible via a zipper. An input port protrudes through a small incision in the fabric at the expected region of the anus, allowing the trainee to pump air into the chamber. The trainee uses a standard hand-held aneroid gauge and bulb insufflator to pump air into the chamber through a plastic tube. The instructor can manipulate a release valve incorporated into the tubing to control the difficulty of maintaining pressure, forcing the trainee to consider causes of air leaks. The chamber contains a spring-loaded piston at one end, allowing the chamber to expand and contract similar to a human colon. A second plastic tube connects the chamber's output port to

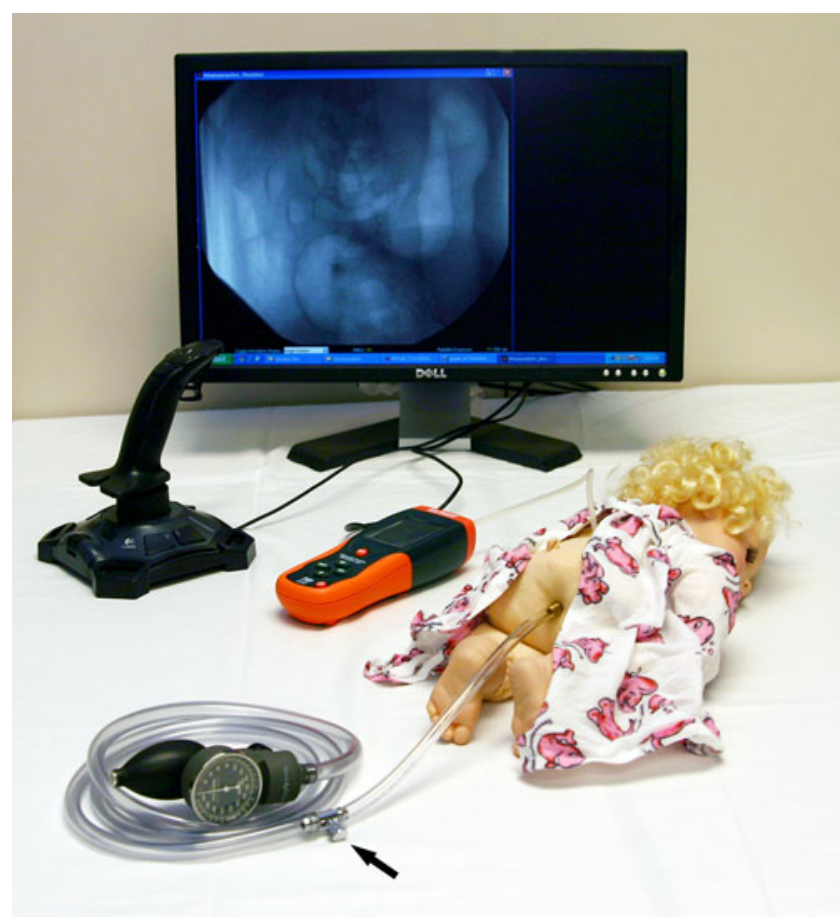

Fig. 1 Plastic tubing with the instructor's external release valve (arrow) connects the aneroid gauge and bulb insufflator to the cylinder within the doll. Additional tubing extends from the doll to a pressure sensor and is transmitted via USB cable to a computer

a pressure sensor (Extech Instruments; Waltham, MA, USA). The sensor measures the air pressure in the chamber every $0.5 \mathrm{~s}$ and delivers the data to a computer over a standard USB interface. The trainee can monitor the pressure inside the chamber by reading the pump's built-in gauge, the pressure sensor screen or the computer screen.

A computer program written in MATLAB (The Mathworks; Natick, MA, USA) records the pressure data and calculates the probability of successfully progressing toward reduction, the probability of regressing, and the probability of perforation during each 0.5 -sec interval (Fig. 2). The probability of progression at each pressure is an estimate based upon review of the literature [5, 8-11] and our personal experience (RSW) with numerous intussusception reductions. The computer generates a random number from 0 to 100 during each 0.5 -sec interval and compares it to the calculated probabilities. If the calculated probability for a particular event is higher than the random number, the simulated procedure progresses, regresses or perforates accordingly. If none of the probabilities is high enough, nothing happens and the computer moves to the next cycle. A flowchart of the computer program can be seen in Fig. 3 .

The trainee using this simulator device can usually reduce the intussusception in about $5 \mathrm{~min}$ if he or she maintains pressure of approximately $120 \mathrm{mmHg}$, and sometimes succeeds with lower pressures. If he or she does 
Fig. 2 Probability of the four possible events as a function of achieved pressure. The probability of perforation increases rapidly after reaching $140 \mathrm{mmHg}$ (diamond). Regression (square) is more likely at low pressures. Early on in the procedure (triangle), probability of progression, or reduction of the intussusceptum, is relatively high, but as the duration of the procedure increases the probability of reduction $(x)$ is much lower

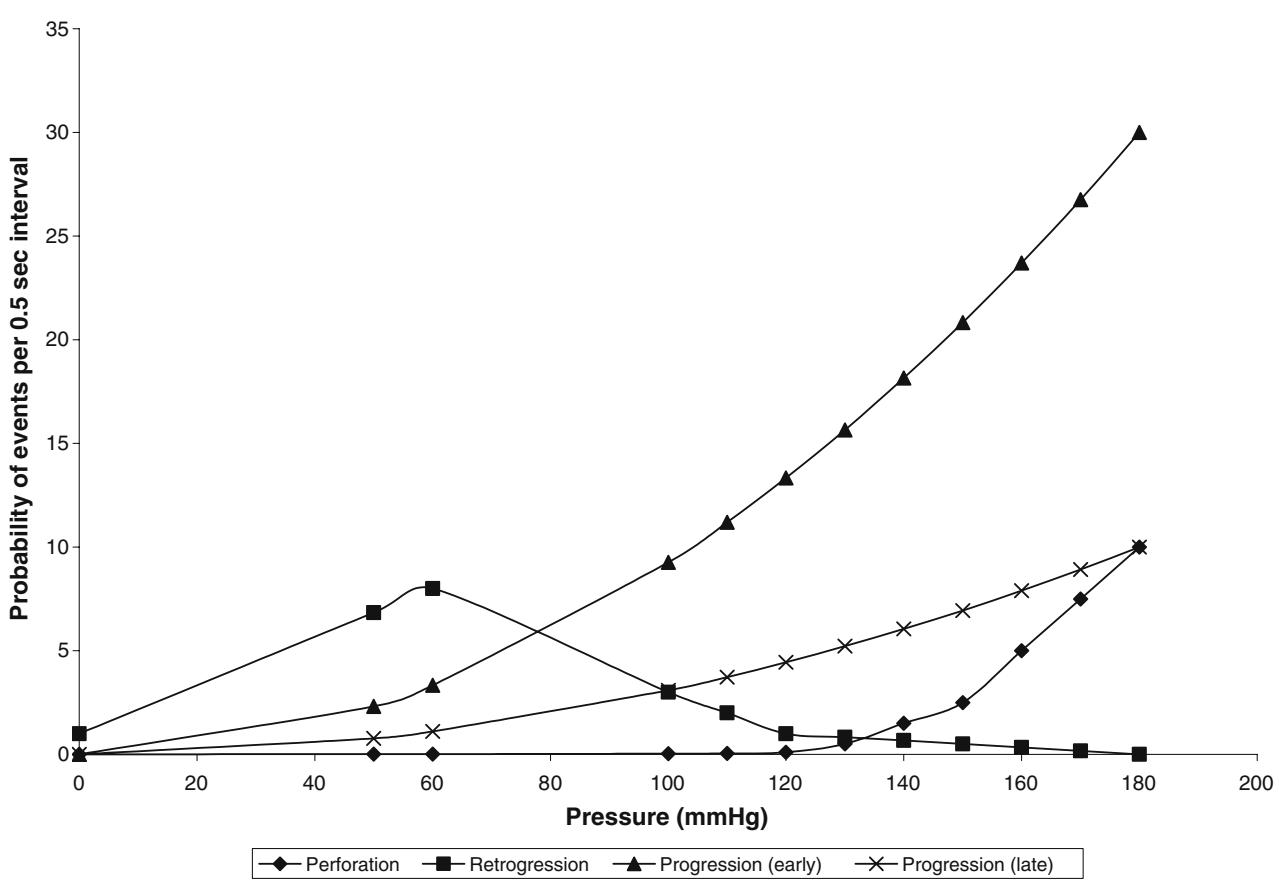

not maintain sufficient pressure, the intussusception often fails to reduce and can regress. If the trainee delivers too much pressure, the chance of perforation increases. The simulation progresses much more quickly than a real-life intussusception reduction in order to limit instructor and trainee fatigue. In addition, because it is critical that a trainee learn to recognize pneumoperitoneum, the probability of perforation is higher than in real life. This gives the trainee opportunities to discuss and simulate management of tension pneumoperitoneum. The simulation progresses quickly and with lower pressures in the earlier stages of reduction, as is common during actual procedures.

The computer program has access to a library of images from actual intussusception reductions, each image corresponding to a particular stage in the reduction, including a preliminary radiograph (Fig. 4), numerous images during the procedure (e.g., Fig. 5) and finally a fluoroscopic image demonstrating complete reduction of the intussusception (Fig. 6). At any point, the user can take a simulated fluoroscopic image via an external joystick trigger that simulates the ergonomics of a fluoroscope. When the trainee presses the trigger, the computer calls up the image corresponding to the current stage of reduction and displays it on the screen. The image remains on the screen until the next time the trainee presses the trigger, simulating imagehold technology used in most modern fluoroscopes.

An inverted, modified radiographic image of a different patient with pneumoperitoneum (Fig. 7) is included in the library, as we have not yet encountered pneumoperitoneum as a direct result of intussusception reduction and therefore can provide no fluoroscopic image of this entity. The image of an abdomen with free air is displayed whenever the probability of perforation is higher than the random number, which is usually at high pressures but occasionally

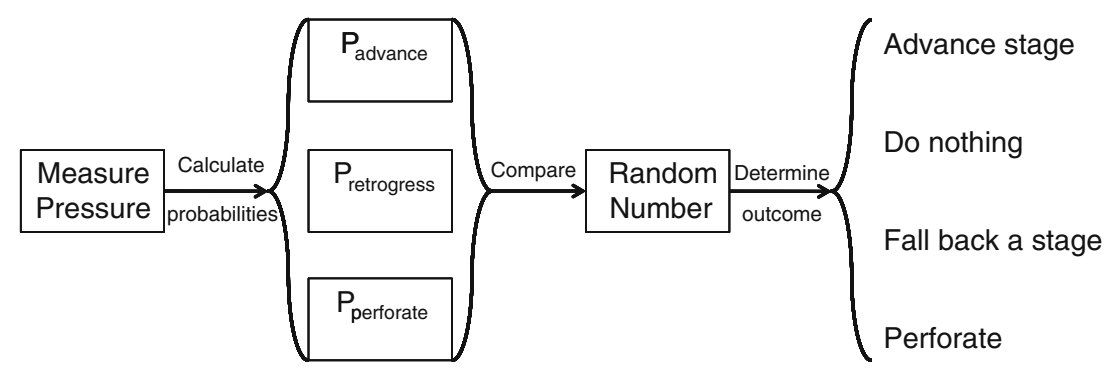

Fig. 3 Flow chart of the MATLAB algorithm. The pressure sensor transmits readings to the computer every $0.5 \mathrm{~s}$. Based on the pressure and the stage of reduction, the computer calculates the probability of advancing to the next stage, retrogressing to the previous stage, and perforating the bowel. The computer then compares these probabilities to a random number and decides what event, if any, will occur. The process then repeats for the next pressure reading 


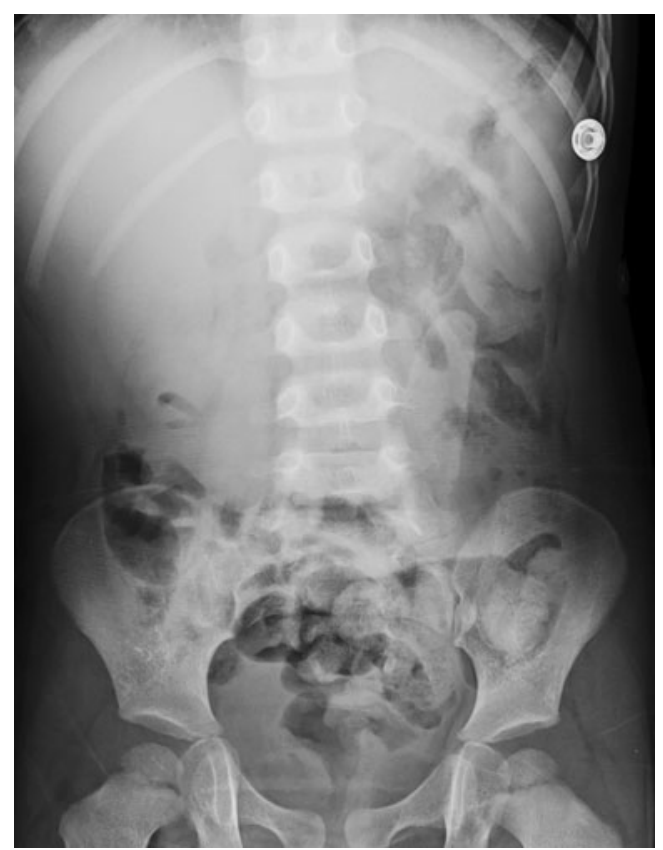

Fig. 4 Supine pre-procedural radiograph of patient with intussusception demonstrates a soft-tissue mass in the right upper abdomen and several mildly dilated loops of bowel

occurs during an otherwise uneventful attempt at reduction. Trainees are expected to recognize the free air and state that they would assess for tension pneumoperitoneum and then simulate treating it by inserting an 18-gauge needle just above the umbilicus. Additionally, the simulator tracks the number of times the trainee triggers the fluoroscope- a proxy for radiation dose-in order to train the user in minimizing radiation dosage to the patient.

Videos of a trainee and instructor reducing an intussusception and of the trainee encountering a perforation are available as electronic supplementary materials 1 and 2 .

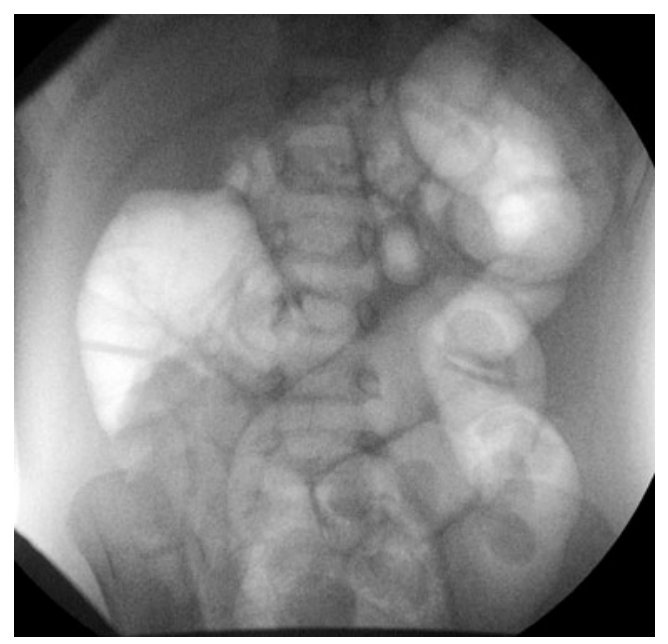

Fig. 5 Fluoroscopic image of patient during a reduction procedure. Air now fills most of the colon, and the soft-tissue mass of the intussusceptum is now in the ascending colon

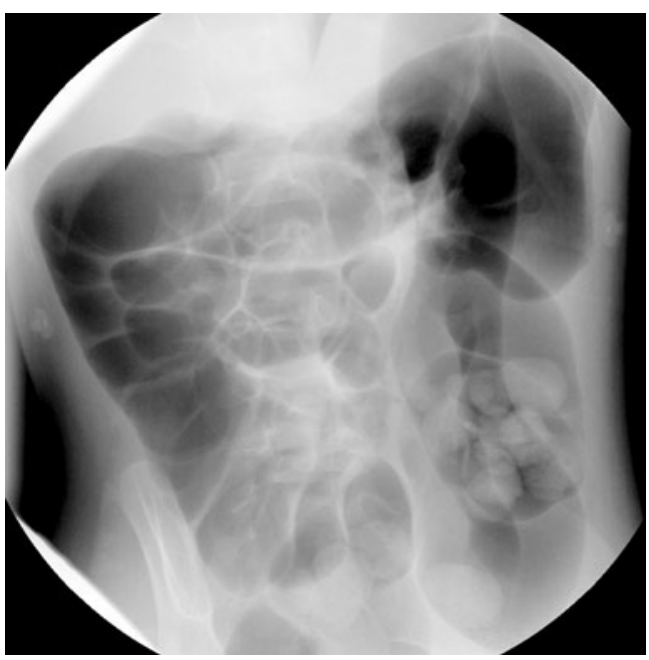

Fig. 6 Fluoroscopic image of a patient immediately after successful reduction of intussusception demonstrates absence of soft-tissue mass and gas filling multiple small bowel loops

\section{Discussion}

George M. Retan described fluoroscopically guided hydrostatic reduction of intussusception in 1927, making it one of the first interventional radiological procedures [12]. It was subsequently popularized by Ravitch and McCune [13] in their experimental study in 1948. Since then, fluoroscopically guided reduction has been considered the initial management of choice for most uncomplicated cases of childhood intussusception. Although US has been used effectively to guide the reduction of intussusception [14, 15], fluoroscopic monitoring is preferred among general and pediatric radiologists in the United States. A survey performed among pediatric radiologists in European teaching hospitals showed that more than $90 \%$ of respondents preferred fluoroscopically guided reduction [16].

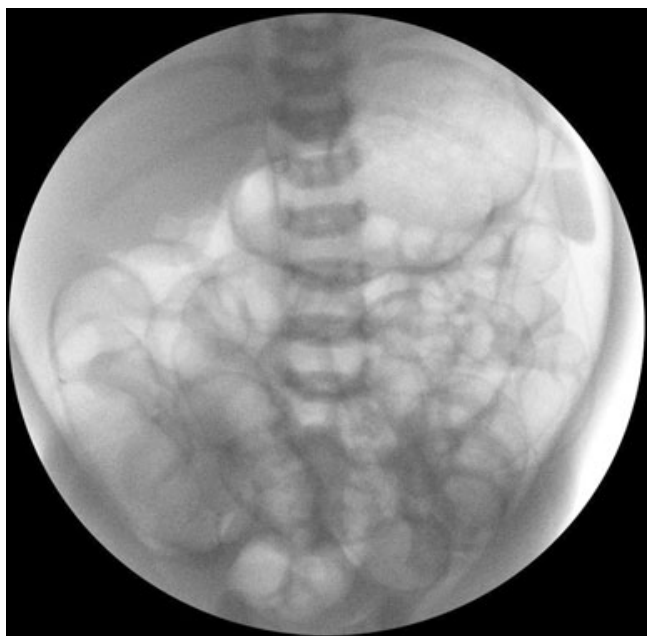

Fig. 7 Inverted modified radiograph of a patient with perforation demonstrates gas outlining bowel loops, liver and spleen 
Both air and liquid are commonly employed for intussusception reduction, but there is a growing trend among pediatric radiologists to shift from hydrostatic to pneumatic reduction $[17,18]$. Air reduction is a quick and clean technique that is easy to learn [17] and is associated with smaller perforations with less fecal peritoneal contamination than liquid enemas [19]. The filling speed with air is also about seven times faster than fluid, facilitating a higher force of reduction [20]. In comparison to fluoroscopic hydrostatic reduction, pneumatic reduction requires less radiation exposure because of shorter fluoroscopic times and lower kilovolt peak and $\mathrm{mA}$ settings $[5,21]$. Because of these advantages coupled with its greater mechanical simplicity, we chose to develop a model that would use the pneumatic technique.

We attempted to simulate experiences that are commonly encountered in intussusception reduction. (1) Air leak occurs when the instructor releases a valve in the tubing. The trainee must recognize that the intussusceptum has stopped reducing or has even regressed and then consider what could be causing the insufflator to cease transmitting pressure. The trainee should carefully assess for pneumoperitoneum, as a sudden pressure drop can occur when the bowel perforates. The trainee might also note a sudden inability to maintain previous pressure levels. (2) The program allows reduction to occur at various speeds, sometimes requiring the trainee to apply pressure for sustained periods without appreciable change in intussusceptum position. Although reduction success correlates with pressure, the use of a random number generator simulates the inherent uncertainty in the process of reducing an intussusception. (3) Some of the images are difficult to interpret because of poor contrast and overlapping air-filled bowel loops, as well as differing orientation depending on whether the patient is supine or prone. The trainee must quickly decipher the images and decide whether reduction is progressing or complete. (4) With excessive pressure - and sometimes with pressures less than $120 \mathrm{mmHg}$ - perforation can occur; the trainee must instantly recognize this complication and discuss treatment of tension pneumoperitoneum. There is no specific trigger for perforation, however, as even at pressures as high as $300 \mathrm{mmHg}$ perforation occurs in only $5 \%$ of patients [22]. Because it is important to recognize this complication, pneumoperitoneum is more common in this simulation than has been reported in actual cases [5]. As in a real intussusception reduction, the trainee must multitask during this procedure by simultaneously controlling the insufflator, monitoring the pressure, ensuring a tight grip on the buttocks, delivering radiation, interpreting fluoroscopic images and springing into action if perforation occurs.

One intrinsic weakness of this device arises from the fact that the statistical likelihood of progression or regression of reduction at multiple specific pressures is apparently not established, with success reported at different pressures that vary among researchers $[5,8-11,21]$. Therefore the values we have chosen are estimates, based on empirical testing of the device, our own experience and literature review. If there is sufficient interest in this device, we will improve it by correlating the patient's clinical presentation with the ease of intussusception reduction. When using this device, the trainee experiences a shorter version of what can be a very lengthy procedure, as we found that both trainees and instructors fatigued when the reduction proceeded at rates often encountered in routine clinical practice. When faced with a real intussusception reduction, the trainee might therefore think that his or her attempt at reduction is unsuccessful if it does not progress as rapidly as it does in this simulator; the instructor must make this clear during the simulation. In addition, the image showing perforation is from a different patient from that in the initial radiographs, making it easy for the resident to recognize that this complication has occurred; however, we have no pneumoperitoneum image occurring during an intussusception reduction in our practice to date. In order to better simulate the actual reduction experience, the device could include a recording of a crying child. Finally, the experience could have been enhanced by allowing the trainee to deliver continuous fluoroscopy (so that they could use excessive radiation more easily, increasing the opportunity for instruction about appropriate radiation delivery), but this proved to be technically challenging. If there is widespread interest in this device, we will work with a commercial partner to make it available and also develop an instructor's manual delineating essential feedback and other points the instructor should emphasize.

Although air reduction has many advantages over liquid, many practicing radiologists were trained with liquid reduction and are uncomfortable with air. This device can help these practitioners transition to using the air reduction technique, enabling them to offer their patients the advantages that air reduction provides. Air reduction is considered easy to learn, but it is difficult to learn in a vacuum.

In conclusion, most radiology residents and general radiologists have limited hands-on experience in reducing intussusception. Although some children with intussusception are treated at pediatric hospitals - and thus by radiologists with a great deal of experience with reduction - many patients present to smaller community facilities, and the success of their reduction depends on a radiologist who may never have performed this procedure, either during training or afterward. Failed reduction in the community often necessitates transfer to a tertiary care facility, and the resulting delay can diminish the likelihood that a repeat attempt at reduction will succeed. Since judgment and 
confidence are best gained and refined by experience, this simulator helps compensate for the rarity of intussusception by simulating important components of air reduction. Residents, fellows and even nervous attending radiologists may, after practicing pneumatic reduction with this device, develop the necessary skills and confidence to successfully reduce their next case of intussusception.

Open Access This article is distributed under the terms of the Creative Commons Attribution Noncommercial License which permits any noncommercial use, distribution, and reproduction in any medium, provided the original author(s) and source are credited.

\section{References}

1. Bruce J, Huh YS, Cooney DR et al (1987) Intussusception: evolution of current management. J Pediatr Gastroenterol Nutr 6:663-674

2. Rennels MB, Parashar UD, Holman RC et al (1998) Lack of an apparent association between intussusception and wild or vaccine rotavirus infection. Pediatr Infect Dis J 17:924-925

3. Swischuk LE, John SD, Swischuk PN (1994) Spontaneous reduction of intussusception: verification with US. Radiology 192:269-271

4. Stein-Wexler R, Bateni C, Gorges SW et al (2010) Radiology residents' experience with intussusception reduction. Pediatr Radiol 40:586-587, Abstract PA127

5. Meyer JS, Dangman BC, Buonomo C et al (1993) Air and liquid contrast agents in the management of intussusception: a controlled randomized trial. Radiology 188:507-511

6. Beasley SW, Myers NA (1998) Intussusception: current views. Pediatr Surg Int 14:157
7. Satava RM (2009) The revolution in medical education - the role of simulation. J Grad Med Educ 1:172-175

8. Stein M, Alton DJ, Daneman A (1992) Pneumatic reduction of intussusception: 5-year experience. Radiology 183:681-684

9. Shiels WE 2nd, Maves CK, Hedlund GL et al (1991) Air enema for diagnosis and reduction of intussusception: clinical experience and pressure correlates. Radiology 181:169-172

10. Kirks DR (1995) Air intussusception reduction: "the winds of change". Pediatr Radiol 25:89-91

11. de Ocampo JF, Phelan E (1989) Gas reduction of intussusception. Pediatr Radiol 19:297-298

12. McDermott VG (1994) Childhood intussusception and approaches to treatment: a historical review. Pediatr Radiol 24:153-155

13. Ravitch MM, McCune RM Jr (1948) Reduction of intussusception by hydrostatic pressure: an experimental study. Bull Johns Hopkins Hosp 82:550-568

14. Riebel TW, Nasir R, Weber K (1993) US-guided hydrostatic reduction of intussusception in children. Radiology 188:513-516

15. Wang GD, Liu SJ (1988) Enema reduction of intussusception by hydrostatic pressure under ultrasound guidance: a report of 377 cases. J Pediatr Surg 23:814-818

16. Schmit P, Rohrschneider WK, Christmann D (1999) Intestinal intussusception survey about diagnostic and nonsurgical therapeutic procedures. Pediatr Radiol 29:752-761

17. Daneman A, Navarro O (2004) Intussusception. Part 2: an update on the evolution of management. Pediatr Radiol 34:97-108

18. Meyer JS (1992) The current radiologic management of intussusception: a survey and review. Pediatr Radiol 22:323-325

19. Daneman A, Alton DJ, Ein $S$ et al (1995) Perforation during attempted intussusception reduction in children - a comparison of perforation with barium and air. Pediatr Radiol 25:81-88

20. Schmitz-Rode T, Müller-Leisse C, Alzen G (1991) Comparative examination of various rectal tubes and contrast media for the reduction of intussusceptions. Pediatr Radiol 21:341-345

21. Phelan E, de Campo JF, Malecky G (1988) Comparison of oxygen and barium reduction of ileocolic intussusception. AJR 150:1349-1352

22. Wilkinson AG (1997) Higher pressure air reduction of intussuception: fewer laparotomies (abstr). Pediat Radiol 27:461 